\title{
Admissible sets for chance-constrained difference inclusions
}

\author{
James Fleming Mark Cannon
}

\begin{abstract}
We study the Maximal Admissible Set (MAS) of a linear difference inclusion under a chance constraint involving random variables with bounded support. After showing that it is difficult to compute this set in general, we suggest inner and outer approximations that can be computed using existing algorithms. The inner approximations are themselves constraint admissible sets and can be represented by a finite number of chance constraints. We give examples to demonstrate the low level of conservatism of these approximations and to illustrate the potential application in Model Predictive Control.
\end{abstract}

\section{INTRODUCTION}

Constraint-admissible sets are an important tool in the study of constrained systems. The theory of admissible sets for linear systems under linear constraints in the absence of uncertainty is given in [1], and more recent publications have considered finding such sets in the presence of disturbances [2], parametric uncertainty [3], for continuous-time systems [4], and for nonlinear systems [5]. An overview of the subject is available in the survey paper [6]. Such sets are often used in order to ensure recursive feasibility in Model Predictive Control [7], [8], and it is stochastic MPC that provides the motivation for this paper: we study admissible sets for polytopic difference inclusions under chance constraints. This is a generalisation of a 'one-step-ahead' chance constraint found in several recent stochastic MPC formulations [9], [10].

Previous attempts to find admissible sets for use in stochastic MPC have centered on finding approximate polytopic sets that are both invariant and respect the chance constraint [9], [11]. We instead find expressions for admissible sets in terms of chance constraints. Hence the sets that we consider may be nonpolyhedral and nonconvex. Methods of computing these sets require chance constrained programming [12], but this is made possible by recent advances in the use of scenario approximations to solve chance-constrained programs [13], [14].

A similar problem is studied in [11] where the probability of the state lying in some polytope at all future time instants is constrained, considering stochastic system dynamics. The authors show that, due to the unboundedness of the uncertainty, applying such constraints robustly for all times does not define an admissible set in general. In contrast we study the more restrictive problem where a probabilistic constraint must be satisfied robustly under dynamics that are polytopic and subject to bounded uncertainty. This can be motivated in MPC applications by the need for recursive feasibility [15]. In addition, in this case the largest set robustly satisfying constraints is an admissible set, overcoming the principal difficulty of [11] that led the authors to consider polytopic approximations to chance-constrained sets. This maximal admissible set includes that given in [15] as a special case.

\section{Mathematical Preliminaries}

We consider dynamics defined by a set-valued function $\Phi: \mathbb{R}^{n} \rightarrow P\left(\mathbb{R}^{n}\right)$. With a slight abuse of notation, we define images and preimages of this function as:

$$
\begin{gathered}
\Phi(\mathcal{A})=\left\{x \in \mathbb{R}^{n}: \exists z \in \mathcal{A}, x \in \Phi(z)\right\} \\
\Phi^{-1}(\mathcal{A})=\left\{x \in \mathbb{R}^{n}: \forall z \in \Phi(x), z \in \mathcal{A}\right\}
\end{gathered}
$$

Such a set-valued function is said to be continuous if the preimage of any closed set is closed and the preimage of any open set is open. The image and preimage operations are inverses of each other, so if $k$-fold repeated images and preimages are denoted by $\Phi^{k}(\mathcal{A})$ and $\Phi^{-k}(\mathcal{A})$ respectively, we have the composition law $\Phi^{p}\left(\Phi^{q}(\mathcal{A})\right)=\Phi^{p+q}(\mathcal{A})$ for all integers $p, q$. These operations distribute over intersections and unions of families of sets in the sense that

$$
\Phi\left(\bigcap \mathcal{A}_{\alpha}\right)=\bigcap \Phi\left(\mathcal{A}_{\alpha}\right), \quad \Phi\left(\bigcup \mathcal{A}_{\alpha}\right) \subseteq \bigcup \Phi\left(\mathcal{A}_{\alpha}\right)
$$

with equivalent relations for unions and intersections of preimages (for preimages equality holds in both cases). The set valued function will be defined as the convex hull of a set of points:

$$
\Phi(x)=\operatorname{Conv}\left(\left\{\Phi_{i}(x): i=1, \ldots, m\right\}\right)
$$

For such functions, the image and preimage operations take the simplified forms,

$$
\Phi(\mathcal{A})=\bigcup_{i=1}^{m} \Phi_{i}(\mathcal{A}), \quad \Phi^{-1}(\mathcal{A})=\bigcap_{i=1}^{m} \Phi_{i}^{-1}(\mathcal{A})
$$

and it is immediate from these expressions that continuity of the functions $\Phi_{i}$ implies continuity of $\Phi$ in the sense defined above. We introduce the following 'multi-index' notation for compositions of the vertex functions $\Phi_{i}(x)$. If $M=\{i, j, \ldots, m\}$ then a finite composition can be written as:

$$
\Phi_{M}(x)=\Phi_{i}\left(\Phi_{j}\left(\ldots \Phi_{m}(x) \ldots\right)\right)
$$

The set of all possible combinations of $k$ indices in the range $1, \ldots, m$ will be denoted $\mathcal{I}_{k}$. For convenience we also define $\Phi_{\emptyset}(x)=x$, that is, setting $M$ as the empty set gives the identity function.

For any sequence of sets $\mathcal{A}_{k}, k=0,1,2, \ldots$ we have the relationship:

$$
\bigcup_{N=0}^{\infty} \bigcap_{k \geq N} \mathcal{A}_{k} \subseteq \bigcap_{N=0}^{\infty} \bigcup_{k \geq N} \mathcal{A}_{k}
$$

The left and right hand sides of this expression are sometimes called the limit inferior and limit superior, in analogy with 
sequences in calculus. If the relation holds with equality, so both sides are equal to a set $\mathcal{A}_{\infty}$, we will define this to be the limit of the sequence and write,

$$
\mathcal{A}_{\infty}=\lim _{k \rightarrow \infty} \mathcal{A}_{k}
$$

which will allow us to investigate the asymptotic properties of set sequences. Finally, the notation $\operatorname{Pr}[E]$ denotes the probability of a condition $E$, so that

$$
\operatorname{Pr}[E]=\mathbb{P}\{\omega \in \Omega: E\}
$$

for $\{\omega \in \Omega: E\} \in \mathcal{F}$, where $\Omega$ denotes some sample space with associated probability measure $\mathbb{P}$ and $\sigma$-algebra $\mathcal{F}$.

\section{PROBLEM Formulation}

Let $k=0,1, \ldots$ be a discrete time variable and $x_{k} \in \mathbb{R}^{n}$ be the state of a system at time $k$. We consider the difference inclusion

$$
x_{k+1} \in \Phi\left(x_{k}\right), \quad k=0,1,2, \ldots
$$

where:

$$
\Phi(x)=\operatorname{Conv}\left\{\left(A^{(i)} x\right), \quad i=1, \ldots, r\right\}
$$

We define a stochastic output variable $y_{k} \in \mathbb{R}^{m}$ by

$$
y_{k}=C_{k} x_{k}+d_{k}
$$

where, for each $k, C_{k}$ and $d_{k}$ are independent realisations of random variables $C(\omega)$ and $d(\omega)$ with $\omega \in \Omega$. We restrict ourselves to considering cases where the support of $C(\omega)$ and $d(\omega)$ is bounded.

Assumption 1. The support of the random variables $C(\omega)$ and $d(\omega)$ is a bounded subset of $\mathbb{R}^{m \times n}$ and $\mathbb{R}^{m}$, respectively.

Consider the closed set

$$
\mathcal{X}_{0}=\left\{x_{k} \in \mathbb{R}^{n} \mid \operatorname{Pr}\left[y_{k} \leq 1\right] \geq p\right\}
$$

which is defined by the chance constraint:

$$
\operatorname{Pr}\left[y_{k} \leq 1\right] \geq p
$$

It is desired to find the largest set of initial states $x_{0}$ such that it is guaranteed that $x_{k} \in \mathcal{X}_{0}$ for all time. In agreement with e.g. [1] this will be termed the Maximal Admissible Set (MAS) of (III.1) respecting (III.5).

Assumption 2. The dynamics (III.2) are such that

$$
\{0\}=\lim _{k \rightarrow \infty} \Phi^{k}\left(\left\{x_{0}\right\}\right)
$$

for any $x_{0} \in \mathbb{R}^{n}$, where 0 denotes the origin.

Asymptotic stability in this sense is equivalent to the joint spectral radius of the matrices $A^{(i)}$ being less than 1 [16]. This stability assumption implies that there exist invariant sets for the dynamics (III.1), that is sets $\mathcal{Z}$ satisfying $\Phi(\mathcal{Z}) \subseteq$ $\mathcal{Z}$. We define an admissible set as an invariant set respecting the constraint (III.5):

Definition 1. For a set-valued function $\Phi$ and constraint set $\mathcal{X}_{0}$, the set $\mathcal{Z}$ is admissible if $\Phi(\mathcal{Z}) \subseteq \mathcal{Z}$ and $\mathcal{Z} \subseteq \mathcal{X}_{0}$.
Suppose that there exist admissible sets $\mathcal{Z}_{i}$ indexed by some $i$. Then the union $\bigcup_{i} \mathcal{Z}_{i}$ is also admissible as $\Phi\left(\bigcup_{i} \mathcal{Z}_{i}\right)=\bigcup_{i} \Phi\left(\mathcal{Z}_{i}\right) \subseteq \bigcup_{i} \mathcal{Z}_{i}$ because $\Phi\left(\mathcal{Z}_{i}\right) \subseteq \mathcal{Z}_{i}$ for all $i$, and $\bigcup_{i} \mathcal{Z}_{i} \subseteq \mathcal{X}_{0}$ because $\mathcal{Z}_{i} \subseteq \mathcal{X}_{0}$ for all $i$. $\mathrm{A}$ similar argument applies to the intersection of a collection of admissible sets. So the class of admissible sets is closed under unions and intersections. This class is nonempty, as Assumption 2 implies that $\{0\}$ is admissible.

Definition 2. A set $\mathcal{Z}_{\max }$ is called the maximal admissible set $(M A S)$ if it is admissible and $\mathcal{Z} \subseteq \mathcal{Z}_{\max }$ for any admissible set $\mathcal{Z}$.

The closure properties under union of the class of admissible sets, combined with the fact that the subset relation ' $\subseteq$ ' gives a partial order on the class, leads directly to a well-known existence and uniqueness result for the MAS.

Proposition 1. Under Assumption 2, the maximal admissible set of (III.1) with constraint (III.5) exists and is unique.

Note that unlike the corresponding problem with linear constraints (see e.g. [16]) we cannot expect the MAS to be convex in general since (III.5) might not be a convex constraint. However if $p \geq 0.5$ various sufficient conditions for convexity of (III.5) can be given; for instance that $C$ has normally-distributed rows and $d$ has a logconcave distribution [17], or if $C$ is a row vector and $[C, 1-d]^{T}$ has a symmetric, logconcave distribution [18]. In such cases it will be shown that the MAS is also convex.

\section{The Maximal Admissible Set}

We use the image and preimage operations to define the sets $\mathcal{X}_{k}$ for any integer $k$ by

$$
\mathcal{X}_{k}=\Phi^{-k}\left(\mathcal{X}_{0}\right)
$$

which implies that $\Phi\left(\mathcal{X}_{k}\right)=\mathcal{X}_{k-1}$ and $\Phi^{-1}\left(\mathcal{X}_{k}\right)=\mathcal{X}_{k+1}$ for all $k$. For $k \geq 0$, this set is given by a finite number of chance constraints:

$\left.\mathcal{X}_{k}=\left\{x_{0} \in \mathbb{R}^{n} \mid \operatorname{Pr}\left[C_{k} \Phi_{M}\left(x_{0}\right)+d_{k}\right) \leq 1\right] \geq p, \quad \forall M \in \mathcal{I}_{k}\right\}$

We further define $\mathcal{S}_{k}$ to be the set of all states in $\mathcal{X}_{j}$ for all $j=0,1, \ldots, k$, that is the set of all states that satisfy the chance constraint up until time $k$.

$$
\mathcal{S}_{k}=\bigcap_{j=0}^{k} \mathcal{X}_{j}=\bigcap_{j=0}^{k} \Phi^{-j}\left(\mathcal{X}_{0}\right)
$$

The following theorem establishes the limit $\mathcal{S}_{\infty}=$ $\lim _{k \rightarrow \infty} \mathcal{S}_{k}$ as the MAS of (III.1) with constraint (III.5).

Theorem 2 (Maximal Admissible Set). $\mathcal{S}_{\infty}$ is the MAS for the dynamics (III.1) and constraint (III.5).

Proof. Using the distribution property of images over intersections, the image of $\mathcal{S}_{\infty}$ under the mapping $\Phi$ is

$$
\Phi\left(\mathcal{S}_{\infty}\right)=\Phi\left(\bigcap_{k=0}^{\infty} \mathcal{X}_{k}\right) \subseteq \bigcap_{k=0}^{\infty} \Phi\left(\mathcal{X}_{k}\right)=\bigcap_{k=-1}^{\infty} \mathcal{X}_{k} \subseteq \mathcal{S}_{\infty}
$$


giving $\Phi\left(\mathcal{S}_{\infty}\right) \subseteq \mathcal{S}_{\infty}$. Because $\mathcal{S}_{\infty} \subseteq \mathcal{X}_{0}$ also, it is admissible.

Now for any admissible set $\mathcal{Z}$ we have $\Phi(\mathcal{Z}) \subseteq \mathcal{Z}$ and $\mathcal{Z} \subseteq \mathcal{X}_{0}$, implying by induction that $\Phi^{k}(\mathcal{Z}) \subseteq \mathcal{X}_{0}$ for $k=$ $1,2, \ldots$. Taking the preimage under $\Phi$ of both sides of this relation $k$ times yields $\mathcal{Z} \subseteq \Phi^{-k}\left(\mathcal{X}_{0}\right)=\mathcal{X}_{k}$ for all $k=$ $1,2, \ldots$. Hence $\mathcal{Z} \subseteq \mathcal{S}_{\infty}$ for any admissible $\mathcal{Z}$ and therefore $\mathcal{S}_{\infty}$ is maximal.

Remark 3. Consider the special case in which the matrix $C=c^{T}$ consists of a single row and is not random. In that case, the constraint defining $\mathcal{X}_{0}$ can be written as

$$
\operatorname{Pr}\left[c^{T} x \leq 1-d\right] \geq p
$$

which is equivalent to

$$
c^{T} x \leq 1-F_{d}^{-1}(p)
$$

where $F_{d}($.$) denotes the distribution function of d$. Then $\mathcal{S}_{\infty}$ is an intersection of polytopes and is polytopic if the spectral radius of $A$ is less than 1 . This case is considered in [15].

We now give sufficient conditions for the convexity and compactness of $\mathcal{S}_{k}$; it will be convex or compact if $\mathcal{X}_{0}$ is convex or compact, respectively. This implies that any condition (such as logconcavity of the distribution of $y_{k}$ ) that implies convexity of the chance constraint (III.5) also implies convexity of the MAS.

Theorem 4 ( $\mathcal{S}_{k}$ inherits convexity, compactness). If $\mathcal{X}_{0}$ is convex or compact then $\mathcal{S}_{k}$ is convex or compact respectively, for all $k=1,2, \ldots, \infty$.

Proof. Taking the preimage of a convex set under an affine mapping preserves convexity [19], and $\mathcal{X}_{k}$ is a finite intersection of such preimages for nonnegative $k$. Hence if $\mathcal{X}_{0}$ is a convex set then so is $\mathcal{X}_{k}$ for any $k>0$. The definition of $\mathcal{S}_{k}$ then implies that $\mathcal{S}_{k}$ and $\mathcal{S}_{\infty}$ are countable intersections of convex sets, therefore they are also convex.

If $\mathcal{X}_{0}$ is compact, then $\mathcal{S}_{k}$ is bounded due to the relation $\mathcal{S}_{k} \subseteq \mathcal{X}_{0}$. Because $\mathcal{X}_{0}$ is closed, continuity of $\Phi$ implies that the set $\mathcal{X}_{k}$ is also closed for any $k$. Hence $\mathcal{S}_{k}$ is closed for any $k$ as it is an intersection of closed sets. Because it is closed and bounded, it is compact.

In the case that $\mathcal{X}_{0}$ is defined by a symmetric linear constraint, it is possible to demonstrate compactness of the MAS under an observability condition (see [16]). We now give a similar condition for the chance-constraint (III.5). Let $C_{1}, C_{2}, \ldots, C_{n}$ and $d_{1}, d_{2}, \ldots, d_{n}$ be independent realisations of $C(\omega)$ and $d(\omega)$ respectively. Then for some $A \in \operatorname{Conv}\left\{A^{(i)}, i=1, \ldots, r\right\}$ define:

$$
O(A)=\left[\begin{array}{c}
C_{1} \\
C_{2} A \\
C_{3} A^{2} \\
\cdots \\
C_{n} A^{n-1}
\end{array}\right], D=\left[\begin{array}{c}
d_{1} \\
d_{2} \\
d_{3} \\
\cdots \\
d_{n}
\end{array}\right]
$$

The following theorem relates the compactness of $\mathcal{S}_{\infty}$ to the rank of the random matrix $O(A)$ in the case of symmetric constraints.
Theorem 5 (Compactness). If $\mathcal{X}_{0}$ is symmetric, and there exists $A \in \operatorname{Conv}\left(A^{(i)}\right)$ such that $O(A)$ has rank $n$ with probability at least $1-p$, then $\mathcal{S}_{k}$ is compact for $k=$ $n-1, n, \ldots, \infty$.

Proof. For any $A \in \operatorname{Conv}\left\{A^{(i)}, i=1, \ldots, r\right\}$, we have the relation $\mathcal{S}_{\infty} \subseteq\left\{x \in \mathbb{R}^{n} \mid \operatorname{Pr}[O(A) x+D \leq 1] \geq p\right\}$. Suppose this latter set is not compact, then due to the symmetry property it is possible to choose an $x$ that lies in the right nullspace of $O(A)$ with probability $p$. But this contradicts the conditions of the theorem. Hence this set is compact and so is $\mathcal{S}_{\infty}$.

Remark 6. When $p=1$, the condition that $O(A)$ has rank $n$ for any given $A$ is equivalent to requiring that, for some $\tilde{C}$ in the support of the random variable $C,(A, \tilde{C})$ is an observable pair. The condition of Theorem 5 can be viewed as a probabilistic generalisation of this observability condition.

The definition of $\mathcal{S}_{\infty}$ contains an infinite number of chance constraints. However the next theorem shows that only a finite number of these constraints are required if the origin lies in the interior of $\mathcal{X}_{0}$.

Theorem 7 (Finite Determination). If $\mathcal{S}_{k}$ is compact for some finite $k$ and $\{0\}$ is in the interior of $\mathcal{X}_{0}$ then $\mathcal{S}_{N}=\mathcal{S}_{\infty}$ for all $N \geq K$, for some sufficiently large but finite $K$.

Proof. Since $\mathcal{S}_{k}$ is compact and $\{0\}$ is in the interior of $\mathcal{X}_{0}$, Assumption 2 implies that there must exist a finite integer $K \geq k$ such that

$$
\Phi^{K+1}\left(\mathcal{S}_{k}\right) \subseteq \mathcal{X}_{0}
$$

Taking the pre-image $K+1$ times gives

$$
\mathcal{S}_{k} \subseteq \Phi^{-K-1}\left(\mathcal{X}_{0}\right)=\mathcal{X}_{K+1}
$$

and since $K \geq k$ implies $\mathcal{S}_{K} \subseteq \mathcal{S}_{k}$, we therefore have $\mathcal{S}_{K} \subseteq$ $\mathcal{X}_{K+1}$. Furthermore $\mathcal{S}_{K+1}=\Phi^{-1}\left(\mathcal{S}_{K}\right) \cap \mathcal{X}_{0}$ by definition, so $\mathcal{S}_{K} \subseteq \mathcal{X}_{K+1}$ implies $\mathcal{S}_{K+1} \subseteq \Phi^{-1}\left(\mathcal{X}_{K+1}\right)=\mathcal{X}_{K+2}$ and hence by induction we obtain

$$
\mathcal{S}_{N} \subseteq \mathcal{X}_{N+1} \quad \forall N \geq K
$$

Finally, by definition we also have that $\mathcal{S}_{N+1}=\mathcal{S}_{N} \cap \mathcal{X}_{N+1}$, so (IV.3) implies $\mathcal{S}_{N+1}=\mathcal{S}_{N}$ for all $N \geq K$, and hence $\mathcal{S}_{N}=\mathcal{S}_{\infty}$ for all $N \geq K$ by induction.

A potential difficulty in applying Theorem 7 is in determining the value of $N$. Consider the problem of deciding if $\mathcal{S}_{N}=\mathcal{S}_{\infty}$ for a given $N$. A positive answer could be given if we were to demonstrate that $\mathcal{S}_{N} \subseteq \mathcal{X}_{N+1}$, which is equivalent to showing $\mathcal{S}_{N} \subseteq\left\{x \in \mathbb{R}^{n} \mid \operatorname{Pr}\left[C \Phi_{M}(x)+d \leq 1\right] \geq p\right\}$ for all $M \in \mathcal{I}_{N+1}$. In the case with $p=1$ this can be verified by solving a linear programming problem. In the chanceconstrained case, this condition is equivalent to the optimal objective of the following optimisation problem being greater than $p$ for all $M \in \mathcal{I}_{N+1}$ :

$$
\begin{array}{lr}
\underset{x}{\operatorname{minimise}} & \operatorname{Pr}\left[C \Phi_{M}(x)+d \leq 1\right] \\
\text { subject to } & x \in \mathcal{S}_{N}
\end{array}
$$


This optimisation is unlikely to be tractable. Indeed in the case that $\mathcal{X}_{0}$ is a convex set the domain $\mathcal{S}_{N}$ will be convex but the objective function is quasiconcave, and so this is not a convex optimisation problem unless the objective is linear (see Remark 3). For this reason, in the next section we turn our attention to finding admissible approximations of the MAS.

\section{ApPROXIMATING THE MAS}

We first note a simple scaling result for invariant sets under the dynamics (III.1) that is required in subsequent proofs.

Lemma 8. If $\Phi(\mathcal{Z}) \subseteq \mathcal{Z}$ for some $\mathcal{Z}$, then $\Phi(\mu \mathcal{Z}) \subseteq \mu \mathcal{Z}$ for any $\mu \geq 0$.

Proof. For any $x \in \mu \mathcal{Z}$ we can write $x / \mu \in \mathcal{Z}$, implying

$$
\operatorname{Conv}\left\{A^{(i)}(x / \mu), \quad i=1, \ldots, r\right\} \subseteq \mathcal{Z}
$$

which is equivalent to:

$$
\operatorname{Conv}\left\{A^{(i)} x, i=1, \ldots, r\right\} \subseteq \mu \mathcal{Z}
$$

proving the Lemma.

We will now construct an approximation to the MAS from admissible sets for a related problem with only linear constraints. Assumption 1 implies that there exists some $C^{(i)}$, $d^{(i)}$ for $i=1,2, \ldots, s$ such that

$$
\operatorname{Pr}\left[\left(C_{k}, d_{k}\right) \in \operatorname{Conv}\left(C^{(i)}, d^{(i)}\right)\right]=1
$$

In that case, we can consider the maximal admissible set of the system (III.1) respecting the linear constraint

$$
\left[C^{(i)}\right]_{j} x_{k}+\left[d^{(i)}\right]_{j} \leq 1
$$

for each $i=1, \ldots, s$ and $j=1, \ldots, m$, where $[C]_{j}$ and $[d]_{j}$ denote the $j$ 'th row of $C$ and $j$ 'th element of $d$. Algorithms for solving this problem can be found in [3] and [16]. We assume that one of these algorithms is available to us and has been used to find an index set defining such an admissible set. Hence, for each $i$ and $j$, define the index set $\mathcal{J}_{j}^{(i)}$ such that

$\mathcal{R}_{j}^{(i)}=\left\{x \in \mathbb{R}^{n} \mid\left[C^{(i)}\right]_{j} \Phi_{M}(x)+\left[d^{(i)}\right]_{j} \leq 1, \quad \forall M \in \mathcal{J}_{j}^{(i)}\right\}$

is an admissible set for the system (III.1) respecting the linear constraint (V.1). We assume that $\emptyset \in \mathcal{J}_{j}^{(i)}$, so that the constraint $\left[C^{(i)}\right]_{j} x+\left[d^{(i)}\right]_{j} \leq 1$ appears in the definition of $\mathcal{R}_{j}^{(i)}$. If we further define an index set $\mathcal{J}=\bigcup_{j=1}^{m} \bigcup_{i=1}^{s} \mathcal{J}_{j}^{(i)}$, then we have the following important property relating events on the sample space $\Omega$.

Lemma 9. For $\mathcal{J}$ as defined,

$$
\begin{gathered}
\left\{\omega \in \Omega \mid C(\omega) \Phi_{M}(x)+d(\omega) \leq 1, \quad \forall M \in \mathcal{J}\right\} \subseteq \\
\left\{\omega \in \Omega \mid C(\omega) \Phi_{M}\left(A^{(i)} x\right)+d(\omega) \leq 1, \quad \forall M \in \mathcal{J}, \quad \forall i\right\}
\end{gathered}
$$

Proof. If, for some $\omega \in \Omega$,

$$
C(\omega) \Phi_{M}(x)+d(\omega) \leq 1, \forall M \in \mathcal{J}
$$

then Assumption 1 implies that we can use $C^{(i)}, d^{(i)}$ to write

$$
\sum_{l=1}^{s} \lambda_{l}\left(C^{(l)} \Phi_{M}(x)+d^{(l)}\right) \leq 1, \quad \forall M \in \mathcal{J}
$$

where $\lambda_{l} \geq 0$ for all $l$ and $\sum_{l=1}^{s} \lambda_{l}=1$. Hence, considering this expression row-wise, there exist some $y_{j}^{(l)} \in \mathbb{R}$ for $l=$ $1, \ldots, s, j=1, \ldots, m$ such that

$$
\left[C^{(l)}\right]_{j} \Phi_{M}(x)+\left[d^{(l)}\right]_{j} \leq y_{j}^{(l)}, \forall M \in \mathcal{J}
$$

with $\sum_{l=1}^{s} \lambda_{l} y_{j}^{(l)} \leq 1$ for all $j$. The definition of $\mathcal{J}$ implies that the constraints

$$
\left[C^{(l)}\right]_{j} \Phi_{M}(x)+\left[d^{(l)}\right]_{j} \leq 1, \quad \forall M \in \mathcal{J}
$$

define an admissible set. Hence, from Lemma 8, the constraints (V.2) define an invariant set and imply:

$$
\left[C^{(l)}\right]_{j} \Phi_{M}\left(A^{(i)} x\right)+\left[d^{(l)}\right]_{j} \leq y_{j}^{(l)}, \quad \forall M \in \mathcal{J}, \quad \forall i
$$

Multiplying by $\lambda_{l}$ and summing gives,

$$
\sum_{l=1}^{s} \lambda_{l}\left(\left[C^{(l)}\right]_{j} \Phi_{M}\left(A^{(i)} x\right)+\left[d^{(l)}\right]_{j}\right) \leq 1, \quad \forall M \in \mathcal{J}, \quad \forall i
$$

which implies (reconstructing the vectors from the rows)

$$
\sum_{l=1}^{s} \lambda_{l}\left(C^{(l)} \Phi_{M}\left(A^{(i)} x\right)+d^{(l)}\right) \leq 1, \quad \forall M \in \mathcal{J}, \quad \forall i
$$

and hence

$$
C(\omega) \Phi_{M}\left(A^{(i)} x\right)+d(\omega) \leq 1, \quad \forall M \in \mathcal{J}, \quad \forall i
$$

completing the proof.

Now consider the two sets:

$$
\begin{aligned}
& \mathcal{P}_{0}=\left\{x \in \mathbb{R}^{n} \mid \operatorname{Pr}\left[C \Phi_{M}(x)+d \leq 1, \quad \forall M \in \mathcal{J}\right] \geq p\right\} \\
& \mathcal{Q}_{0}=\left\{x \in \mathbb{R}^{n} \mid \operatorname{Pr}\left[C \Phi_{M}(x)+d \leq 1\right] \geq p, \quad \forall M \in \mathcal{J}\right\}
\end{aligned}
$$

We further define $\mathcal{P}_{k}$ and $\mathcal{Q}_{k}$ recursively by taking preimages and intersections with $\mathcal{X}_{0}$ :

$$
\mathcal{P}_{k+1}=\Phi^{-1}\left(\mathcal{P}_{k}\right) \cap \mathcal{X}_{0}, \quad \mathcal{Q}_{k+1}=\Phi^{-1}\left(\mathcal{Q}_{k}\right) \cap \mathcal{X}_{0}
$$

It will be shown that both of these sequences of sets converge at least asymptotically to $\mathcal{S}_{\infty}$ as $k \rightarrow \infty$. It can be verified by induction that the solutions of these recurrences for $k \geq 1$ are

$$
\mathcal{P}_{k}=\Phi^{-k}\left(\mathcal{P}_{0}\right) \cap \mathcal{S}_{k-1}, \quad \mathcal{Q}_{k}=\Phi^{-k}\left(\mathcal{Q}_{0}\right) \cap \mathcal{S}_{k-1},
$$

and hence both of these sets can be specified in terms of a finite number of chance constraints. The definition of $\mathcal{Q}_{k}$ immediately implies that it is a superset of the MAS for all $k$, since it is defined by a subset of the constraints defining $\mathcal{S}_{\infty}$. We will show that any $\mathcal{P}_{k}$ is an admissible set for the system (III.1) under constraint (III.5).

Theorem 10 ( $\mathcal{P}_{0}$ is admissible). The set $\mathcal{P}_{0}$ is admissible.

Proof. We first show $\Phi\left(\mathcal{P}_{0}\right) \subseteq \mathcal{P}_{0}$. Choose some $x \in \mathcal{P}_{0}$, then by definition we have

$$
\operatorname{Pr}\left[C \Phi_{M}(x)+d \leq 1, \quad \forall M \in \mathcal{J}\right] \geq p
$$


and hence there exists $\mathcal{E} \subseteq \Omega$ with $\mathbb{P}(\mathcal{E}) \geq p$ such that, for all $\omega \in \mathcal{E}$ :

$$
C(\omega) \Phi_{M}(x)+d(\omega) \leq 1, \quad \forall M \in \mathcal{J}
$$

Lemma 9 now implies that, for all $\omega \in \mathcal{E}$,

$$
C(\omega) \Phi_{M}\left(A^{(i)} x\right)+d(\omega) \leq 1, \quad \forall M \in \mathcal{J}, \quad \forall i
$$

and hence:

$$
\operatorname{Pr}\left[C \Phi_{M}\left(A^{(i)} x\right)+d \leq 1, \quad \forall M \in \mathcal{J}\right] \geq p
$$

We have therefore shown that for any $x \in \mathcal{P}_{0}$ we also have $x \in \Phi^{-1}\left(\mathcal{P}_{0}\right)$. This implies $\mathcal{P}_{0} \subseteq \Phi^{-1}\left(\mathcal{P}_{0}\right)$ and thus $\Phi\left(\mathcal{P}_{0}\right) \subseteq \mathcal{P}_{0}$ after taking the image. As we required $\emptyset \in \mathcal{J}_{j}^{(i)}$ in the definition of the index set, we also have $\emptyset \in \mathcal{J}$ so that the definition of $\mathcal{P}_{0}$ includes the constraint

$$
\operatorname{Pr}[C x+d \leq 1] \geq p
$$

implying that $\mathcal{P}_{0} \subseteq \mathcal{X}_{0}$ and hence that $\mathcal{P}_{0}$ is admissible.

We may now show that $\mathcal{P}_{k}, k=1,2, \ldots$ forms a sequence of admissible sets of increasing size and complexity.

Theorem 11 ( $\mathcal{P}_{k}$ is admissible). $\mathcal{P}_{k}$ is admissible for all $k$. Moreover, $\mathcal{P}_{k} \subseteq \mathcal{P}_{k+1}$ for all $k$.

Proof. We prove that if $\mathcal{P}_{k}$ is admissible, then so is $\mathcal{P}_{k+1}$. If $\mathcal{P}_{k}$ is admissible, then $\Phi\left(\mathcal{P}_{k}\right) \subseteq \mathcal{P}_{k}$. Taking the preimage on both sides and then an intersection with $\mathcal{X}_{0}$ yields

$$
\mathcal{P}_{k} \cap \mathcal{X}_{0} \subseteq \Phi^{-1}\left(\mathcal{P}_{k}\right) \cap \mathcal{X}_{0}
$$

and noting that the left-hand side is $\mathcal{P}_{k}$ and the right-hand side $\mathcal{P}_{k+1}$ implies $\mathcal{P}_{k} \subseteq \mathcal{P}_{k+1}$. Now considering the image $\Phi\left(\mathcal{P}_{k+1}\right)$,

$$
\Phi\left(\mathcal{P}_{k+1}\right)=\Phi\left(\Phi^{-1}\left(\mathcal{P}_{k}\right) \cap \mathcal{X}_{0}\right) \subseteq \mathcal{P}_{k} \cap \Phi\left(\mathcal{X}_{0}\right) \subseteq \mathcal{P}_{k},
$$

and hence $\Phi\left(\mathcal{P}_{k+1}\right) \subseteq \mathcal{P}_{k+1}$. Since $\mathcal{P}_{k+1} \subseteq \mathcal{X}_{0}$ from (V.3), $\mathcal{P}_{k+1}$ is admissible. As we also know that $\mathcal{P}_{0}$ is admissible from Lemma 10, the result follows by induction.

Admissibility of $\mathcal{P}_{k}$ implies that $\mathcal{P}_{k} \subseteq \mathcal{S}_{\infty}$. In particular, this means that $\mathcal{P}_{k}$ will be compact if $\mathcal{S}_{\infty}$ is. Expression (V.4) can be used to investigate convergence of $\mathcal{Q}_{k}$ and $\mathcal{P}_{k}$.

Theorem 12 (Convergence to $\mathcal{S}_{\infty}$ ). As $k \rightarrow \infty, \mathcal{P}_{k} \rightarrow \mathcal{S}_{\infty}$ and $\mathcal{Q}_{k} \rightarrow \mathcal{S}_{\infty}$. Furthermore, if there exists $k$ such that $\mathcal{S}_{k}$ compact and $\{0\}$ is in the interior of $\mathcal{X}_{0}$, then $\mathcal{P}_{N}=\mathcal{S}_{\infty}$ and $\mathcal{Q}_{N}=\mathcal{S}_{\infty}$ for all $N \geq K$, for some sufficiently large but finite $K$.

Proof. Taking the set-theoretic limit of (V.4) gives:

$$
\lim _{k \rightarrow \infty} \mathcal{P}_{k}=\lim _{k \rightarrow \infty} \Phi^{-k}\left(\mathcal{P}_{0}\right) \cap \lim _{k \rightarrow \infty} \mathcal{S}_{k}
$$

The definition of $\mathcal{S}_{k}$ and $\mathcal{S}_{\infty}$ implies $\lim _{k \rightarrow \infty} \mathcal{S}_{k}=\mathcal{S}_{\infty}$. Since $\mathcal{P}_{0}$ is admissible, $\{0\} \subseteq \mathcal{P}_{0}$ and Assumption 2 implies $\lim _{k \rightarrow \infty} \Phi^{-k}\left(\mathcal{P}_{0}\right) \supseteq \lim _{k \rightarrow \infty} \Phi^{-k}(\{0\})=\mathbb{R}^{n}$. Hence

$$
\lim _{k \rightarrow \infty} \mathcal{P}_{k}=\mathbb{R}^{n} \cap \mathcal{S}_{\infty}=\mathcal{S}_{\infty}
$$

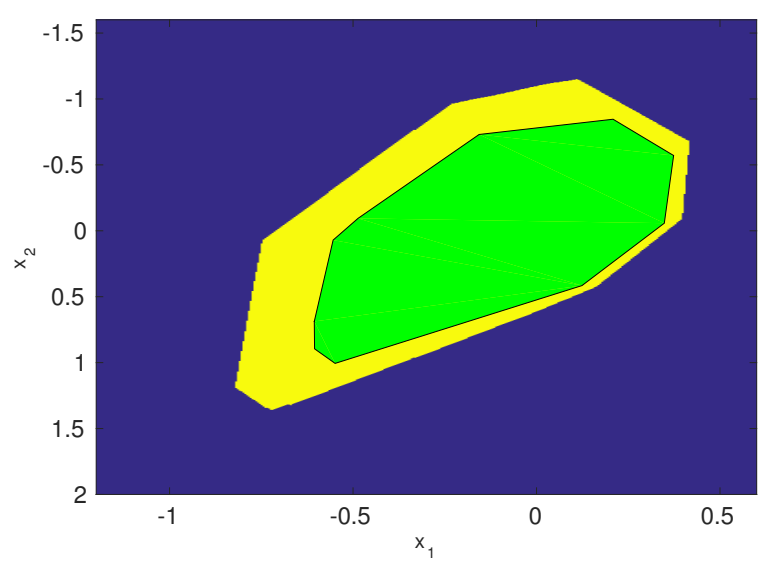

Fig. 1. The set $P_{0}$ (yellow) compared to the robust MAS (green) for Example 1. $Q_{0}$ is indistinguishable from $P_{0}$ at this scale.

Further, if $\mathcal{S}_{k}$ is compact and $\{0\}$ is in the interior of $\mathcal{X}_{0}$, then $\mathcal{S}_{N}=\mathcal{S}_{\infty}$ for some finite $N$ by Theorem 7. Then

$$
\mathcal{P}_{N}=\Phi^{-N}\left(\mathcal{P}_{0}\right) \cap \mathcal{S}_{\infty}
$$

and Assumption 1 then implies that $\mathcal{S}_{\infty} \subseteq \Phi^{-N}\left(\mathcal{P}_{0}\right)$ if $N$ is sufficiently large, leading to $\mathcal{P}_{N}=\mathcal{S}_{\infty}$. The proof for $\mathcal{Q}_{k}$ is nearly identical, so we omit it for brevity.

A consequence of Theorem 12 is that $\mathcal{P}_{k}$ and $\mathcal{Q}_{k}$ give successively better inner and outer approximations to the MAS as $k$ is increased. In some applications, it may be desirable to find out how conservative $x \in \mathcal{P}_{k}$ is compared to $x \in \mathcal{S}_{\infty}$. To this end, the solution to $\min f(x)$ s.t. $x \in \mathcal{S}_{\infty}$ is lower bounded by the solution to $\min f(x)$ s.t. $x \in \mathcal{Q}_{k}$. The domain of this optimisation is convex since, by (V.4), $\mathcal{Q}_{k}$ is a finite intersection of convex sets.

\section{NUMERICAL EXAMPLES}

\section{A. Example 1 - Approximating the MAS}

We considered the random matrix $A=\sum_{i=1}^{3} A^{(i)} q_{i}$, with $q_{i} \in[0,1]$ uniformly distributed random variables for $i=1,2,3$. Hence $q_{i}$ lies in the unit cube in $\mathbb{R}^{3}$, and $A$ is contained in a corresponding set defined by the convex hull of 8 points. Matrices $A^{(i)}$ and $F$ were chosen as:

$$
\begin{array}{ll}
A^{(1)}=\left[\begin{array}{ll}
0.37 & 0.21 \\
0.75 & 0.65
\end{array}\right], & A^{(2)}=\left[\begin{array}{cc}
0.32 & 0.03 \\
1.36 & 0.75
\end{array}\right] \\
A^{(3)}=\left[\begin{array}{cc}
0.43 & -0.33 \\
1.05 & 0.79
\end{array}\right], \quad F=\left[\begin{array}{cc}
1 & 1 \\
-1 & 2 \\
1.5 & -1 \\
-1.5 & 0
\end{array}\right]
\end{array}
$$

The MAS of this system under a robust one-step ahead state constraint $F A x \leq 1, \forall A$ was computed. This can be compared with the sets $\mathcal{P}_{0}$ and $\mathcal{Q}_{0}$ from the previous section for the chance constraint $\operatorname{Pr}[F A x \leq 1] \geq p$ with $p=0.9$, which in this case are defined by 10 joint or marginal chance constraints respectively (i.e. $|\mathcal{J}|=10$ ). These sets were 


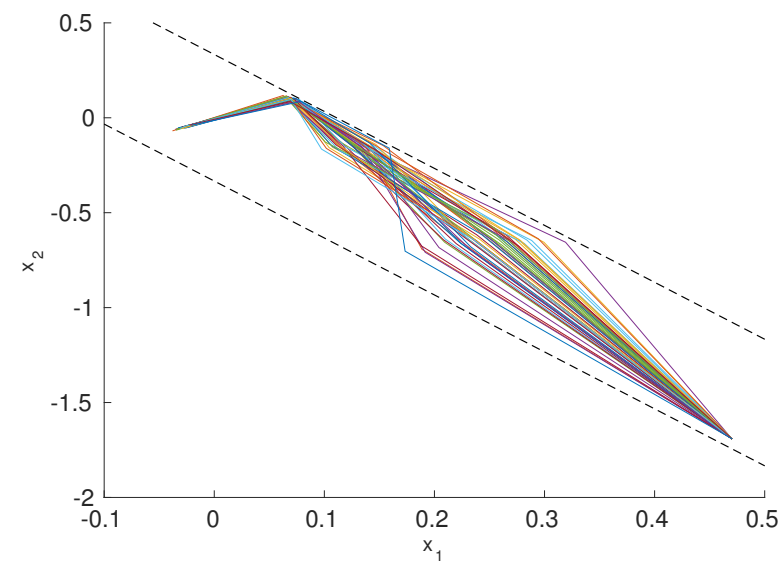

Fig. 2. MPC trajectories using robust MAS, with state constraint

plotted by gridding over the state space $\mathbb{R}^{2}$ and sampling $A$ to evaluate $\operatorname{Pr}[F A x \leq 1]$ at each point (Figure 1). The volume of $\mathcal{P}_{0}$ is $24.6 \%$ larger than the MAS for the robust constraint. Moreover, the difference between $\mathcal{P}_{0}$ and $\mathcal{Q}_{0}$ is indistinguishable at the scale of Figure 1. Since the MAS $\mathcal{S}_{\infty}$ is a subset of $\mathcal{Q}_{0}$, this implies that the admissible set $\mathcal{P}_{0}$ is a very good approximation to $\mathcal{S}_{\infty}$ for this example.

\section{B. Example 2 - Model Predictive Control}

The system and constraint in this section are taken from [10], which considers the control of a stable system $x_{k+1}=$ $\Phi x_{k}+B c_{k}$ where the matrices $\Phi$ and $B$ are uncertain. An alternative to the MPC algorithm given there is to augment the state with control parameters $\underline{c}_{k}=\left[c_{k}^{T} c_{k+1}^{T} \ldots c_{k+N}^{T}\right]^{T}$ to give the dynamics in an autonomous form:

$$
\left[\begin{array}{c}
x_{k+1} \\
\underline{c}_{k+1}
\end{array}\right]=\left[\begin{array}{cc}
\Phi & B E \\
0 & T
\end{array}\right]\left[\begin{array}{l}
x_{k} \\
\underline{c}_{k}
\end{array}\right]
$$

Here $E$ and $T$ are the matrices such that $c_{k}=E \underline{c}_{k}$ and $\underline{c}_{k+1}=T \underline{c}_{k}$. Following [7], It is possible to form a recursively feasible MPC controller by minimising a quadratic cost function subject to the constraint that $\left(x_{k}, \underline{c}_{k}\right)$ lies within an admissible set of (VI.1).

This approach was tested using the cost function, system and constraints of [10] with $p=0.7$. The state evolution when a robust MAS is used is shown in Figure 2. Alternatively, Figure 3 shows the state evolution when $\mathcal{P}_{0}$ is used with chance constraints handled through a scenario approximation of 40 samples. Using $\mathcal{P}_{0}$ leads to a much less conservative control than the robust MAS, with $75 \%$ of the closed loop trajectories satisfying the constraint. Although it is effective, for long prediction horizons this method may produce many chance constraints (e.g. $|\mathcal{J}|=118$ for $N=5$ here), so the approach of [10] is often more competitive due to the use of a prediction tube.

\section{REFERENCES}

[1] E. G. Gilbert and K. T. Tan, "Linear systems with state and control constraints: The theory and application of maximal output admissible sets," Automatic Control, IEEE Transactions on, vol. 36, no. 9, pp. 1008-1020, 1991.

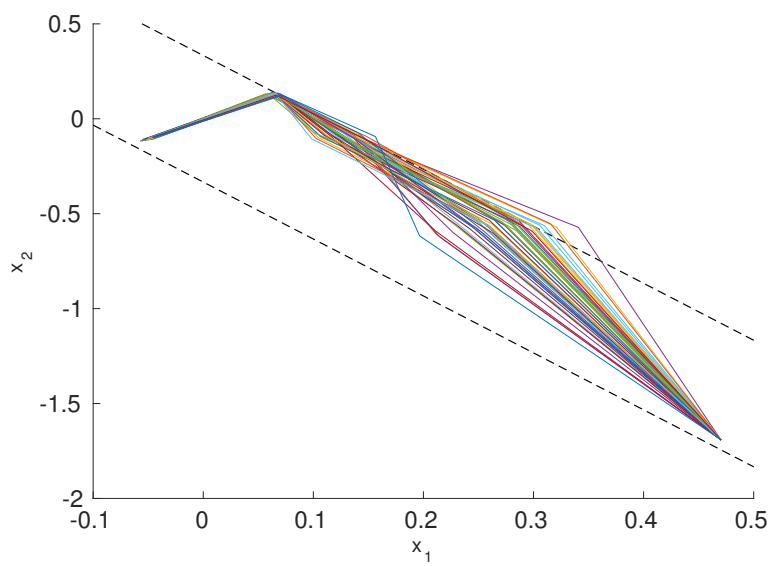

Fig. 3. MPC using $P_{0}$ as admissible set, with state constraint

[2] I. Kolmanovsky and E. G. Gilbert, "Theory and computation of disturbance invariant sets for discrete-time linear systems," Mathematical Problems in Engineering, vol. 4, no. 4, pp. 317-367, 1998.

[3] B. Pluymers, J. Rossiter, J. Suykens, and B. De Moor, "The efficient computation of polyhedral invariant sets for linear systems with polytopic uncertainty," in American Control Conference, 2005. Proceedings of the 2005. IEEE, 2005, pp. 804-809.

[4] F. Blanchini and S. Miani, "Constrained stabilization of continuoustime linear systems," Systems \& control letters, vol. 28, no. 2, pp. 95-102, 1996.

[5] E. C. Kerrigan and J. M. Maciejowski, "Invariant sets for constrained nonlinear discrete-time systems with application to feasibility in model predictive control," in Decision and Control, 2000. Proceedings of the 39th IEEE Conference on, vol. 5. IEEE, 2000, pp. 4951-4956.

[6] F. Blanchini, "Survey paper: Set invariance in control," Automatica (Journal of IFAC), vol. 35, no. 11, pp. 1747-1767, 1999.

[7] B. Pluymers, J. Rossiter, J. Suykens, and B. De Moor, "A simple algorithm for robust mpc," in Proceedings of the IFAC World Congress, 2005

[8] J. A. Rossiter, Model-based predictive control: a practical approach CRC press, 2013

[9] M. Evans, M. Cannon, and B. Kouvaritakis, "Robust and stochastic linear MPC for systems subject to multiplicative uncertainty," in 4th IFAC Nonlinear Model Predictive Control Conference, vol. 4, no. 1, 2012, pp. 335-341.

[10] J. Fleming, M. Cannon, and B. Kouvaritakis, "Stochastic tube MPC for LPV systems with probabilistic set inclusion conditions," in 53rd IEEE Conference on Decision and Control, Los Angeles, USA, 2014

[11] C. Wang, C.-J. Ong, and M. Sim, "Linear systems with chance constraints: Constraint-admissible set and applications in predictive control," in Decision and Control, 2009 held jointly with the 2009 28th Chinese Control Conference. CDC/CCC 2009. Proceedings of the 48th IEEE Conference on. IEEE, 2009, pp. 2875-2880.

[12] A. Prékopa, Stochastic programming. Springer Science \& Business Media, 2013, vol. 324

[13] G. C. Calafiore, "Random convex programs," SIAM Journal on Optimization, vol. 20, no. 6, pp. 3427-3464, 2010.

[14] M. C. Campi and S. Garatti, "A sampling-and-discarding approach to chance-constrained optimization: feasibility and optimality," Journal of Optimization Theory and Applications, vol. 148, no. 2, pp. $257-$ 280, 2011.

[15] B. Kouvaritakis, M. Cannon, S. V. Raković, and Q. Cheng, "Explicit use of probabilistic distributions in linear predictive control," Automatica, vol. 46, no. 10, pp. 1719-1724, 2010

[16] F. Blanchini and S. Miani, Set-theoretic methods in control. Springer, 2007

[17] A. Prékopa, Stochastic programming. Kluwer Academic Publishers, 1995.

[18] C. M. Lagoa, X. Li, and M. Sznaier, "Probabilistically constrained linear programs and risk-adjusted controller design," SIAM Journal on Optimization, vol. 15, no. 3, pp. 938-951, 2005.

[19] S. Boyd and L. Vandenberghe, Convex optimization, 2004. 